Capítulo 7 Dificultades que se presentan en estudiantes al cambiar inesperadamente su ambiente de aprendizaje de presencial a virtual

Chapter 7 Difficulties that occur in students when changing unexpectedly their learning environment from face-to-face to virtual

COTERO-MORENO, Karina Margarita†* \& RODRÍGUEZ-JIMÉNEZ, Liza Mayela

Universidad de Guadalajara, Sistema de Universidad Virtual

ID $1^{\text {er }}$ Autor: Karina Margarita, Cotero-Moreno / ORC ID: 0000-0002-8389-5334, CVU CONACYT ID: 850498

ID $1^{\text {er }}$ Coautor: Liza Mayela, Rodríguez-Jiménez / ORC ID: 0000-0002-9084-3831, CVU CONACYT ID: 396313

DOI: $10.35429 /$ H.2020.7.122.138

K. Cotero, L. Rodríguez

kcotero@udgvirtual.udg.m

A. Marroquín, J. Olivares, L. Cruz y A. Bautista. (Coord) Educación. Handbooks-@ECORFAN-Mexico, Querétaro, 2020. 


\title{
Resumen
}

Este capítulo muestra datos acerca de las dificultades presentadas por estudiantes del calendario 2020A, quienes debido a la contingencia de salud causado por COVID19, tuvieron que migrar de la modalidad de Aprendizaje Presencial a la Modalidad de Aprendizaje Virtual con la finalidad de concluir sus programas académicos en tiempo y forma, establecidos por la Secretaría de Educación Pública. Para lograr el objetivo de mostrar tales dificultades, primero se seleccionaron y conceptualizaron las habilidades necesarias para incursionar en el Aprendizaje Virtual. Después, se seleccionó una muestra aleatoria de diferentes niveles académicos, a quienes se les aplicó la encuesta, de la cual se utilizaron diez ítems para llevar a cabo esta investigación. Enseguida presentamos los resultados y análisis relacionados para documentar este estudio. Finalmente, concluimos este capítulo con una breve reflexión acerca de las competencias más relevantes requeridas por los estudiantes, que les permitan desempeñarse exitosamente en un entorno de Aprendizaje Virtual, logrando así los objetivos establecidos en su programa académico.

\section{Competencias, Aprendizaje Virtual, Aprendizaje Presencial}

\begin{abstract}
This chapter shows data about the difficulties which are externalized by students of the 2020A calendar, who in order to conclude their academic programs in a timely manner established by the Secretary of Public Education, they had to migrate from face-to-face learning modality to virtual modality due to the health contingency caused by the COVID19 outbreak. To achieve the objective of showing such difficulties, first the skills needed to dabble in virtual learning were selected and conceptualized. Subsequently, a random sample was selected from different academic levels to which a survey was applied, to carry out this research only ten items from this survey were used. We present the results and related analysis to keep record of this research. Finally, we conclude this chapter with a brief reflection about the most relevant skills required by the students that allow them a successful virtual academic performance thus achieving the objectives established in their academic program.
\end{abstract}

\section{Skills, e-learning, classroom learning}

\section{Introducción}

Este capítulo tiene como objetivo identificar las competencias necesarias para que un estudiante, al cambiar inesperadamente de una modalidad de aprendizaje presencial a una virtual, se desempeñe exitosamente.

En la actualidad estamos viviendo una crisis mundial debido a la aparición del SARS-COV2. Surgió en China en diciembre pasado, provocando una enfermedad llamada COVID-19, que se extendió por el mundo y fue declarada pandemia global por la Organización Mundial de la Salud. Los coronavirus humanos se transmiten de una persona infectada a otra(s) a través de las gotículas que expulsa un enfermo al toser o estornudar, al tocar o estrechar la mano de una persona enferma, al tocar un objeto o superficie contaminados con el virus y llevarse las manos a boca, nariz u ojos.

Una vez declarada Pandemia los Gobiernos de los países tuvieron que tomar medidas preventivas, en el caso de México a mediados del mes de marzo el subsecretario de Prevención y Promoción de la Salud, Hugo López-Gatell Ramírez, presentó dos acuerdos que fueron aprobados por unanimidad:

"Las acciones de prevención y control, incluidas las de sana distancia (distanciamiento social) serán definidas por la Secretaría de Salud y en consenso con las dependencias involucradas en su aplicación, las fechas de inicio y término de las mismas, así como extensión territorial de aplicación.

La Secretaría de Salud pondrá a disposición de todas las dependencias, el Lineamiento de Sana Distancia (Distanciamiento Social), el cual describe de manera específica las actividades a llevar a cabo en los diferentes ámbitos de actuación (ejemplo: laboral, escolar, comercios, eventos masivos, reclusorios, asilos, templos, entre otros)". 
El Diario Oficial de la Federación estableció en el acuerdo número 02/03/20, la suspensión de clases en las escuelas de educación preescolar, primaria, secundaria, normal y demás, para la formación de maestros de educación básica del Sistema Educativo Nacional, así como aquellas de los tipos medio superior y superior dependientes de la Secretaría de Educación Pública. ” (https://dof.gob.mx).

Una vez tomadas estas medidas, y con la finalidad de seguir cumpliendo la normatividad establecida por la Ley General de Educación, en su capítulo II del ejercicio al derecho a la educación, artículo 5; una de las estrategias propuestas por las instituciones educativas fue dar continuidad al calendario escolar 2020A por medios virtuales, en donde los estudiantes que cursan sus estudios en la modalidad presencial, se vieron en la necesidad de incursionar en la modalidad de aprendizaje virtual.

Si bien es cierto que la educación es un derecho constitucional, y que por dicha razón se establecieron nuevos métodos para dar continuidad a la formación académica de los ciudadanos, también es cierto que los estudiantes se han enfrentado a una dinámica totalmente desconocida, y a un modelo pedagógico diferente al que estaban acostumbrados.

Hoy en día estamos inmersos en una nueva sociedad del conocimiento, en la cual, el surgimiento de las Tecnologías de la Información y la Comunicación ( TIC ) ha generado la necesidad a nivel mundial, de incursionar en esta nueva forma de interacción y generación de conocimiento en los diversos ámbitos, dentro de los cuales el educativo no ha sido una excepción.

Esta nueva forma de pensamiento, convierte a la tecnología en el punto de contacto para gestionar la socialización, sin embargo, nos surge una duda al respecto, el saber si realmente la sociedad en su conjunto está preparada para esta nueva forma de socializar, en su estudio Aguilar (2008) menciona que: El individualismo en red no significa que la sociedad esté constituida por individuos aislados, representa un nuevo modelo social de organización. Los individuos se agrupan voluntariamente de acuerdo con intereses, valores, afinidades, y proyectos comunes. Aunque la solidez de estas relaciones puede ser más efímera en el tiempo que las anteriores, no significa que sean menos intensas o productivas. Por el contrario, ésta parece ser una de las ventajas de las relaciones actuales. Sin embargo, como establece Castells (Castells, 2001c:149), el costo de la individualización aún no se puede determinar pues el proceso es demasiado joven. ( p. 44).

Es bien sabido que el aprendizaje por medios virtuales no es nuevo, y que cada vez más personas apuestan por esta vía para adquirir conocimiento, aún se cuenta con procesos de educación presenciales, en donde Romero-Mayoral, Jesús; García-Domínguez, Melchor; Roca-González, Cristina; Sanjuán Hernán Pérez, Alejandra; Pulido-Alonso, Antonio (2014) mencionan que:

Contar con que emisor (profesor) y receptor (estudiante) se encuentren físicamente en un mismo lugar y a una misma hora (clase), otorga elementos que dan la posibilidad de retroalimentación y de autorregulación, los cuales son muy valiosos para este tipo de actividad. Un profesor puede saber cuando sus estudiantes no han comprendido un tema (retroalimentación), entonces, lo puede reelaborar y expresar de manera diferente (autorregulación) para que sus estudiantes entiendan la temática, comprobando de nuevo el efecto obtenido (control). ( p. 174).

Por la necesidad de seguir proporcionando educación para que los estudiantes de la modalidad presencial continuarán con su proceso educativo sin verse afectados por los lineamientos de sana distancia, la irrupción de la modalidad virtual fue inminente, la cual tuvo que adaptarse de la mejor forma posible, para dar continuidad al proceso educativo del calendario 2020A.

Encontramos entonces que la educación presencial difiere de la educación virtual en varios aspectos, entre los cuales identificamos que uno de los más importantes son las competencias que el estudiante requiere para lograr su objetivo de aprendizaje, es aquí donde éste es separado de su entorno educativo de manera abrupta, surgiendo una serie de conflictos que no le permiten adaptarse a una nueva metodología de aprendizaje.

Por lo anterior, y con la finalidad de obtener información respecto a esta problemática presentada, e identificar cuáles serían las competencias ideales que requiere un estudiante para integrarse a la modalidad de aprendizaje virtual por primera ocasión, realizamos una investigación con aquellos estudiantes que se han visto afectados en este proceso de cambio. 


\subsection{Descripción del método}

Se utilizó el enfoque cualitativo con técnica de la encuesta, debido a que se consideró esencial obtener datos que proporcionen la percepción y/u opinión de los estudiantes, respecto a su incursión al aprendizaje virtual por primera vez.

El instrumento aplicado consta de 21 ítems, del cual sólo se utilizaron 10, ya que son los más relevantes para el desarrollo de esta investigación, las preguntas de la 3 a la 10 son de opción múltiple, lo cual nos permitió obtener un rango mayor de respuestas por estudiante.

La encuesta se aplicó por medios virtuales a una muestra de 84 estudiantes de diversos niveles educativos; Educación Superior, Bachillerato y algunos de Educación Básica. Se les envió vía correo electrónico la liga de acceso al instrumento, el cual se desarrolló con herramientas web, que nos permitieron obtener valores estadísticos de las respuestas obtenidas.

\subsection{Análisis de las variables}

En esta investigación se identificó que la variable dependiente es: dificultades que enfrentan los estudiantes al no contar con las competencias requeridas al cambiar de modalidad de aprendizaje presencial a la modalidad virtual por causas de fuerza mayor.

Las variables independientes con las que trabajamos son:

- Administración del tiempo

- Responsabilidad

- Competencias digitales

- Autogestión

La problemática generada por parte de los estudiantes que cursan la modalidad presencial, surge primordialmente por las dificultades para adaptarse a la dinámica del nuevo modelo de aprendizaje, considerando entonces la siguiente hipótesis: los estudiantes no cuentan con las competencias necesarias que les permitan desempeñarse adecuadamente en un ambiente de aprendizaje virtual.

En este capítulo se mostrarán los resultados obtenidos de la aplicación de un instrumento de evaluación de competencias, así como las propuestas de mejora. En dicho instrumento, se detectó que los estudiantes carecen de las competencias con las que deberían contar para desempeñarse adecuadamente en la modalidad virtual, dichas competencias son las variables independientes en esta investigación, y se explican a continuación:

\section{Administración del Tiempo}

La administración del tiempo es una de las competencias que requiere desarrollar un estudiante que incursiona en la modalidad de aprendizaje virtual, debido a que no ve al docente explicando el tema de forma presencial, sino que el docente quizá se conecte un instante o suba un video en donde explique el trabajo que se debe de realizar; el estudiante es entonces el único responsable de cumplir en tiempo y forma con las actividades solicitadas, y para realizarlas si no comprende las instrucciones establecidas, tendrá que investigar en medios confiables, para entonces poder elaborar la actividad adecuadamente y este proceso le requerirá más tiempo de lo habitual.

Otra cosa importante a considerar para una buena administración del tiempo es que un estudiante por lo general está inscrito en un mínimo de 3 materias de manera simultánea lo cual puede resultar más complicado si no administra adecuadamente su tiempo para el seguimiento de cada una. 
La capacidad de administrar el tiempo nos lleva al éxito o al fracaso, el tiempo no se recupera por lo tanto es importante gestionarlo adecuadamente y no desperdiciarlo, cuanto mejor se utilice el tiempo más se logrará y mayor será la recompensa.

Brian Tracy (2016) menciona en su libro las 4 D para la efectividad de una buena administración del tiempo:" La primera D es el deseo: debes de tener un intenso y apasionado deseo de controlar el tiempo y lograr la máxima eficacia, La segunda D: debes tomar la decisión de practicar buenas técnicas de gestión del tiempo hasta que se conviertan en un hábito. La tercera D significa determinación debes estar dispuesto a persistir ante todas las tentaciones en contra hasta que te hayas convertido en un gestor eficaz del tiempo. La cuarta $\mathrm{D}$ es la disciplina debes disciplinarte para hacer la gestión del tiempo una práctica permanente, la disciplina efectiva es la voluntad de obligarse a hacer lo que sabes que tienes que hacer, cuando debas hacerlo te apetezca o no".

\section{Responsabilidad}

La responsabilidad es un valor determinante en el individuo, según la Real Academia Española señala que la responsabilidad es la capacidad que existe en todo sujeto activo de derecho para reconocer y aceptar las consecuencias de un hecho realizado libremente.

En el caso de esta investigación la responsabilidad es un valor relevante, ya que los docentes no están supervisando a sus estudiantes de manera presencial, por lo que deben confiar en que el estudiante tendrá la capacidad de realizar y enfrentar los retos que se le presentan con el fin de lograr la entrega de sus actividades; el estudiante por su parte tiene la responsabilidad del cumplimiento de las mismas, acorde a las instrucciones y lineamientos especificados.

Esta es una de las dificultades de estudiar en línea, prácticamente el estudiante tiene toda la responsabilidad de lograr las competencias requeridas, si él está en negación o no le interesa realizar las actividades no lo hará, porque no cuenta con la guía(profesor) que la educación presencial le provee. Aquí es donde el docente puede motivar e incentivar al estudiante, sembrar o ayudar a desarrollar de alguna forma este valor o cualidad.

\section{Competencias Digitales}

En la actualidad, las tecnologías de la Información y la Comunicación (TIC) se han convertido en herramientas indispensables en la práctica académica pero existe mucha confusión al respecto, el estudiante piensa que saber utilizar un teléfono inteligente, o buscar información en la computadora usando los buscadores como google es la forma adecuada del proceso de aprendizaje en línea, lo cual se afirma cuando por parte de la institución, se implementan programas pedagógicos en donde en la mayoría de las actividades se solicita leer y hacer resumen como producto a entregar.

Beatriz Zempoalteca, et.al (2017) mencionan en su investigación que una de las mayores potencialidades de las TIC radica en el desarrollo de competencias tecnológicas, digitales e informacionales, pues su uso por los docentes facilita la generación de dichas competencias en los estudiantes. Actualmente, el nuevo paradigma educativo está centrado en el estudiante y el aprendizaje, lo que implica cambios en las tareas profesionales del docente; por ello, se debe adaptar su perfil profesional a las nuevas exigencias del contexto, en especial por las derivadas de las TIC (Pozos \& Mas, 2010)

Ramírez y Casillas (2014) Dicen que el desarrollo de nuevos estilos de enseñanza-aprendizaje a través de las tecnologías trae implicaciones de carácter económico que podrían afectar el capital monetario del estudiante, por lo que eventualmente, adquirirá algún tipo de dispositivo digital si es que desea aprovechar al máximo su aprendizaje.

Actualmente, no sólo los estudiantes han sido afectados por la contingencia presentada a causa de COVID 19, la afectación ha recaído en diversos ámbitos, el laboral es uno de ellos. Por lo que consecuentemente, los integrantes de una familia harán mayor uso de su conexión a internet que les permita realizar sus actividades respectivamente, lo cual se convierte en un problema, ya que el rendimiento de la conexión con la que se cuenta se verá disminuido. 
Entonces, para la elaboración de las tareas, conectarse con sus maestros, buscar información, entre otras de las actividades que requiere realizar el estudiante, es posible que no siempre lo logre ya que depende de un ancho de banda compartido y limitado, lo que generará impaciencia y frustración en caso de no lograr los objetivos solicitados en la actividad.

Otro punto importante a considerar es la implementación variada de herramientas tecnológicas por parte de la institución, los docentes deben considerar el uso de plataformas y aplicaciones tecnológicas, con las que están familiarizados los estudiantes, o en su defecto, que sean intuitivas y fácil de utilizar desde la primera vez que el estudiante tiene esta experiencia. En este punto, quizás se torna algo complicada la parte de licenciamiento, ya que no todas las instituciones cuentan con los recursos necesarios para pagar por sistemas tecnológicos ad hoc a sus necesidades, pero por otro lado, existe también software libre que una vez investigado y analizado, podría cubrir algunas o la mayoría de las características de aprendizaje buscadas en el entorno educativo de cada institución.

\section{Autogestión}

Ponce (2016) Menciona que el término "autogestión" cobra cada vez mayor fuerza en el discurso educativo bajo la forma de desiderátum o aspiración; los modelos pedagógicos de las instituciones apuestan por la autogestión como motor de cambio del sistema tradicional: enseñar ya no es lo primordial, sino impulsar al que aprende desde el reconocimiento de sus propias capacidades. La incorporación de herramientas tecnológicas en los procesos de formación acentúa el deseo de que el estudiante regule la manera en que se apropia del conocimiento y le encuentra relevancia.

Se asume que el alumno autogestivo es un promotor de sus propios aprendizajes y más que eso: un evaluador de sus procesos de apropiación, con lo que también se pone de manifiesto su capacidad meta cognitiva. En teoría, se trata de un estudiante muy diferente al de la modalidad tradicional, mucho más participativo, propositivo, proactivo, crítico, reflexivo, organizado, exigente y colaborador.

Los estudiantes que cursan la modalidad presencial no son autogestivos porque están acostumbrados a que el docente explique los temas en clase, les indique la o las actividades a elaborar, proporcionándoles el material de apoyo, y al mismo tiempo resuelve dudas que los estudiantes tienen en el transcurso del desarrollo de su actividad.

En la modalidad virtual es muy diferente, el profesor casi nunca esta de manera presencial, las instrucciones y actividades se encuentran hospedadas en una plataforma, la tarea del estudiante es leer la actividad y buscar en internet o en el apartado de recursos la bibliografía que requiere para hacerla, él solo elabora su tarea y la envía para su revisión nadie le retroalimenta antes de que la envié, una vez enviada el estudiante espera la respuesta del docente.

\subsection{Resultados}

De los cuestionarios enviados, resultó una muestra real final de 84 encuestas respondidas por estudiantes de diferentes niveles educativos.

Gráfico 7.1 Grado Académico

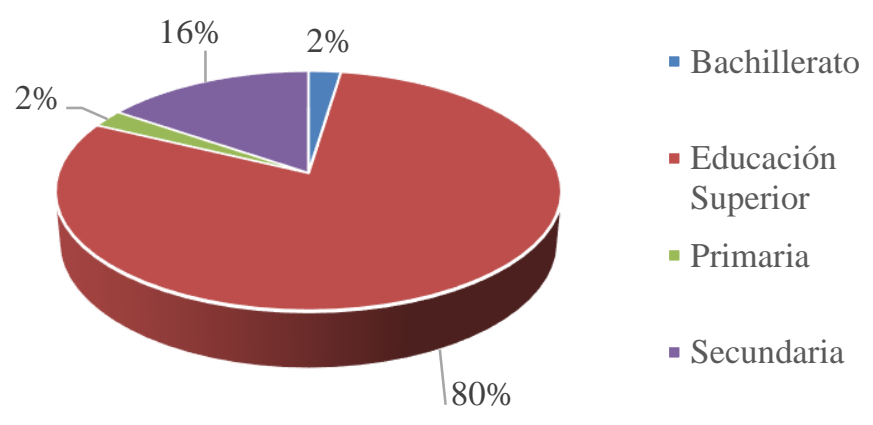

Fuente de consulta: "Elaboración Propia" 
Los datos presentados en el gráfico 7.1, muestran el porcentaje de estudiantes que contestaron el instrumento respecto al nivel educativo que cursan actualmente: Educación Superior 80\%; Bachillerato $2 \%$; Secundaria 16\%; Primaria 2\%. Es claro detectar que un mayor porcentaje de los encuestados son de educación superior, por lo que podríamos considerar que son personas que a lo largo de su proceso educativo hasta este momento ha sido por la modalidad presencial.

Gráfico 7.2 Características de los encuestados

Todos lo niveles educativos Educación Superior
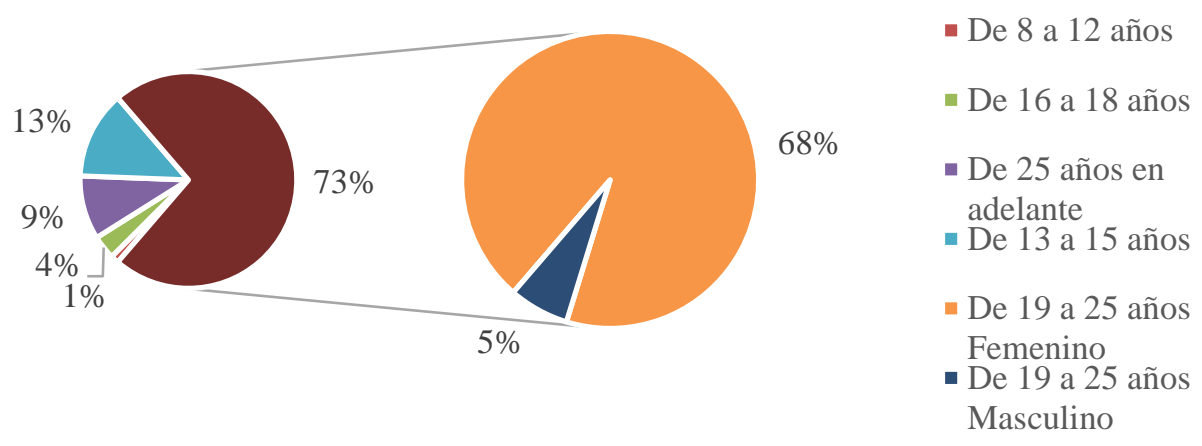

Fuente de consulta: "Elaboración Propia"

En el gráfico 7.2, se identifica que un $73 \%$ de los encuestados cursan el nivel superior, los cuales tienen edades entre 19 y 25 años; esta información nos lleva a la premisa de que la edad es un factor importante en los estudiantes de la modalidad virtual, ya que como lo indica Moreno y Cárdenas López, indican que a decir de García Aretio (2006), alguno de los puntos necesarios de resaltar de las características que definen a un estudiante de la modalidad virtual son:

- Los estudiantes en educación a distancia forman grupos heterogéneos en edad, intereses, ocupación, motivaciones, experiencias y aspiraciones.

- Suelen ser personas que trabajan y que destinan además un tiempo para estudiar.

- Las preocupaciones de los alumnos a distancia se centran en su trabajo, bienestar familiar, ascenso social y laboral, autoestima, etc.

- Los estudiantes a distancia presentan una mayor responsabilidad sobre sus actos; desarrollan un sentimiento de culpa ante expectativas no logradas.

- Tienen que trabajar cuando los otros descansan; el estudio no es su única obligación.

- Reciben el conocimiento aunado a las experiencias y conocimientos anteriores, propiciando que lo cuestionen o no lo asimilen de inmediato. (s/p) 


\section{Gráfico 7.3 Conectividad}

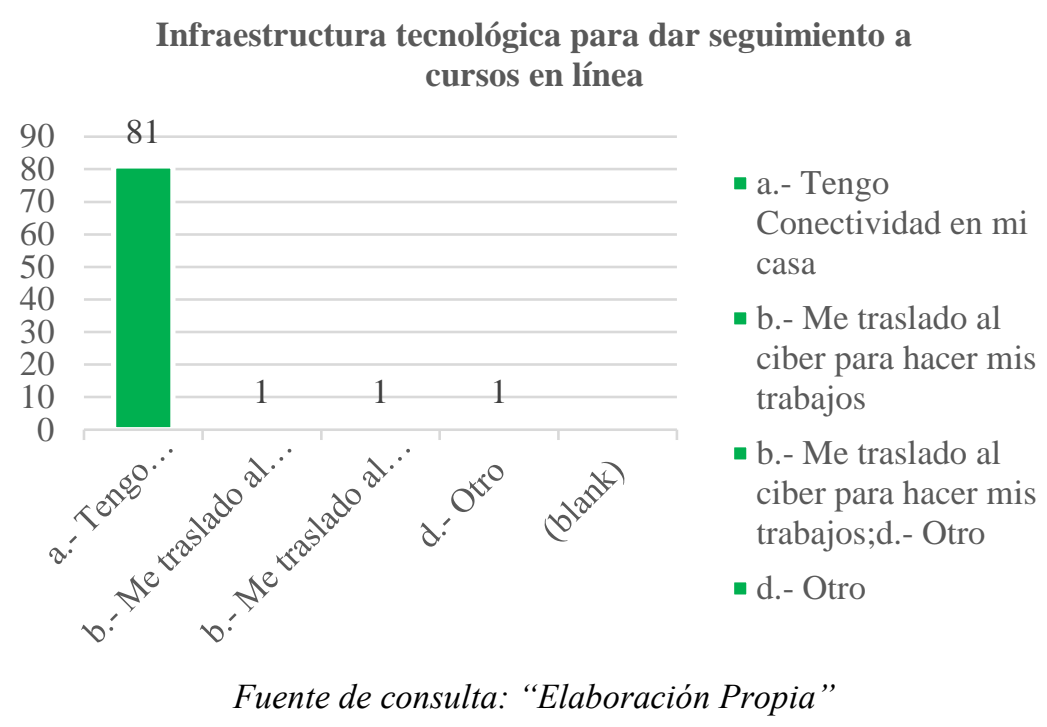

En el gráfico 7.3, los datos obtenidos muestran que el mayor porcentaje de los estudiantes encuestados no tuvieron complicaciones respecto al cambio de modelo educativo en cuanto a la conectividad, ya que 81 estudiantes de 84, afirmaron contar con servicio de conexión a internet en sus hogares, por lo que el medio para comunicarse con sus compañeros y profesor no ha sido un obstáculo en su proceso educativo.

Gráfico 7.4 Impacto de la Modalidad Virtual en los estudiantes

Nivel de afección a nivel personal de los estudiantes al cambio de modalidad de aprendizaje Manera Positiva
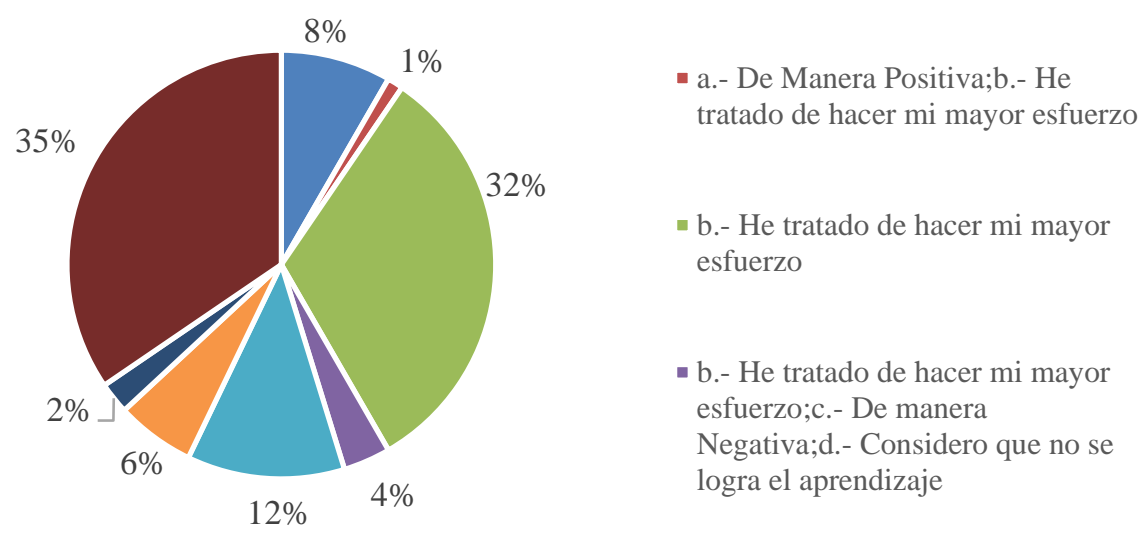

Fuente de consulta: "Elaboración Propia"

Los datos presentados En el gráfico 7.4, muestran que el 35\% de los estudiantes coinciden en que no se logra el aprendizaje por medio de la modalidad virtual; mientras que el $32 \%$ de estudiantes menciona que han intentado hacer su mayor esfuerzo respecto al impacto que les causó el cambio abrupto de modalidad de aprendizaje; el $12 \%$ de los estudiantes identifican que han tratado de hacer su mayor esfuerzo, sumado a que consideran que no se logra el aprendizaje; y el $4 \%$ de los estudiantes consideran que en suma que han tratado de hacer su mayor esfuerzo+impacto negativo + no se logra el aprendizaje. Continuando con este mismo gráfico, al $6 \%$ de los estudiantes, les ha afectado de manera negativa el cambio de modalidad de aprendizaje; el $2 \%$ coinciden en que les ha afectado de manera negativa, sumado a que consideran que no se logra el aprendizaje.

Dentro de los mismos resultados, 76 estudiantes de una muestra de 84, no se ven favorecidos por el cambio en la modalidad de aprendizaje establecido. 
Siguiendo con los resultados del gráfico 7.4, el 1\% de los estudiantes, indican que hacen su mayor esfuerzo, pero afirman que sí les ha impactado de manera positiva; y finalizamos con solo el $8 \%$, quienes consideran que el cambio de aprendizaje de la modalidad presencial a la modalidad virtual ha impactado de manera positiva.

Gráfico 7.5 Horas dedicadas al estudio en Modalidad Virtual

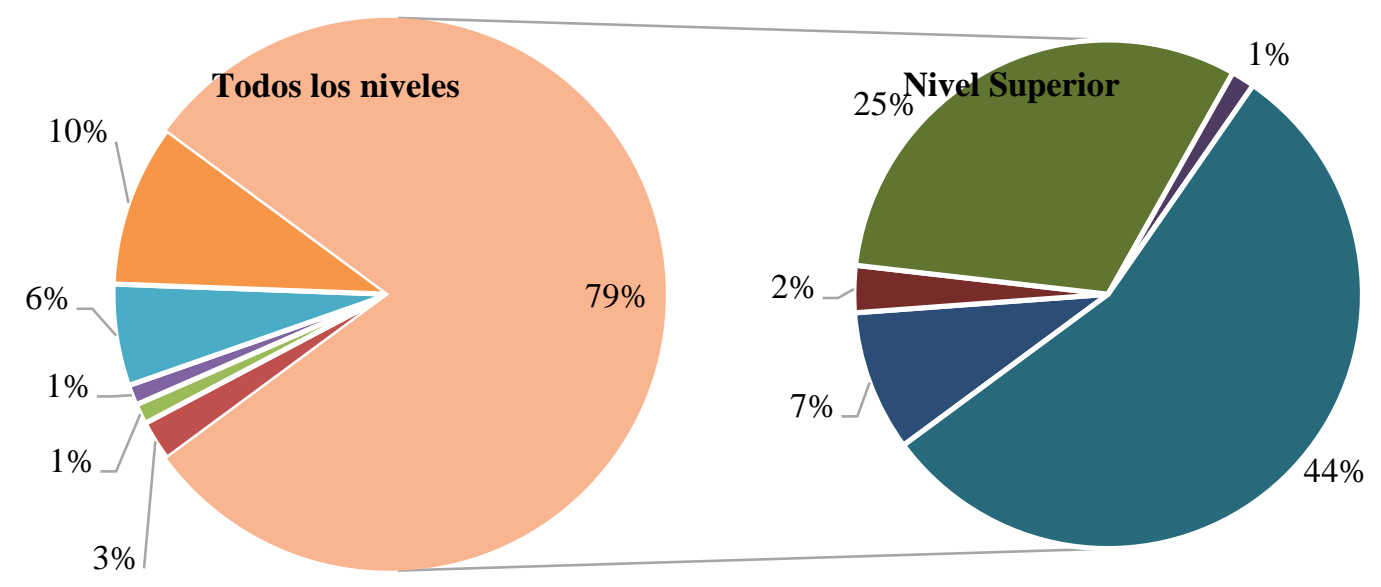

- Primaria b.- 2 a 3 horas al dia

- Bachillerato b.- 2 a 3 horas al dia

- Bachillerato c.- 4 horas o mas

- Secundaria b.- 2 a 3 horas al dia

- Secundaria c.- 4 horas o mas

- Educación Superior a.- 1 hora al dia

- Educación Superior a.- 1 hora al dia;b.- 2 a 3 horas al dia

- Educación Superior b.- 2 a 3 horas al dia

\section{Fuente de consulta: "Elaboración Propia"}

En el gráfico 7.5, se identificó que el 7\% de los estudiantes le dedica 1 hora al día a sus estudios; mientras que el $2 \%$ varía entre 1 hora al día y 2 a 3 horas al día; el $1 \%$ oscila entre 2 a 3 horas al día y 4 horas o más; el 35\% le dedica 2 a 3 horas al día; y finalmente el $55 \%$ le dedica 4 horas o más a sus estudios.

Considerando entonces los resultados anteriores, encontramos, que del $78 \%$ de los estudiantes de Educación Superior, el 25\% de los estudiantes dedican de 2 a 3 horas al día y el 44\%, 4 horas o más al día a sus estudios.

El sistema de educación presencial consta de 6 horas al día de clases, de lunes a viernes, y además es necesario que el estudiante dedique tiempo extra para realizar actividades fuera del horario escolar. A diferencia del sistema de aprendizaje presencial en el aprendizaje virtual, el estudiantes organiza su tiempo para realizar ambas actividades, quienes regularmente dedican de 3 a 4 horas al día a sus actividades educativas, ya que quienes incursionan en la modalidad de aprendizaje virtual, son personas que no cuentan con el tiempo suficiente para dedicarse al estudio de tiempo completo.

Si consideramos entonces que los estudiantes encuestados cuentan con la mayor parte de su tiempo durante el día para llevar a cabo a sus actividades, entonces, consideramos que en lo que se requiere trabajar, es en la administración del tiempo que dedican para su aprendizaje en esta nueva modalidad, que si bien no la eligieron, es importante que desarrollen las 4 D, de Brian Tracy(2006), deseo, decisión, determinación, y disciplina, a fin de optimizar y eficientar el tiempo para mejorar su rendimiento. 
Gráfico 7.6 Ventajas del uso de videoconferencias para el desarrollo de las competencias

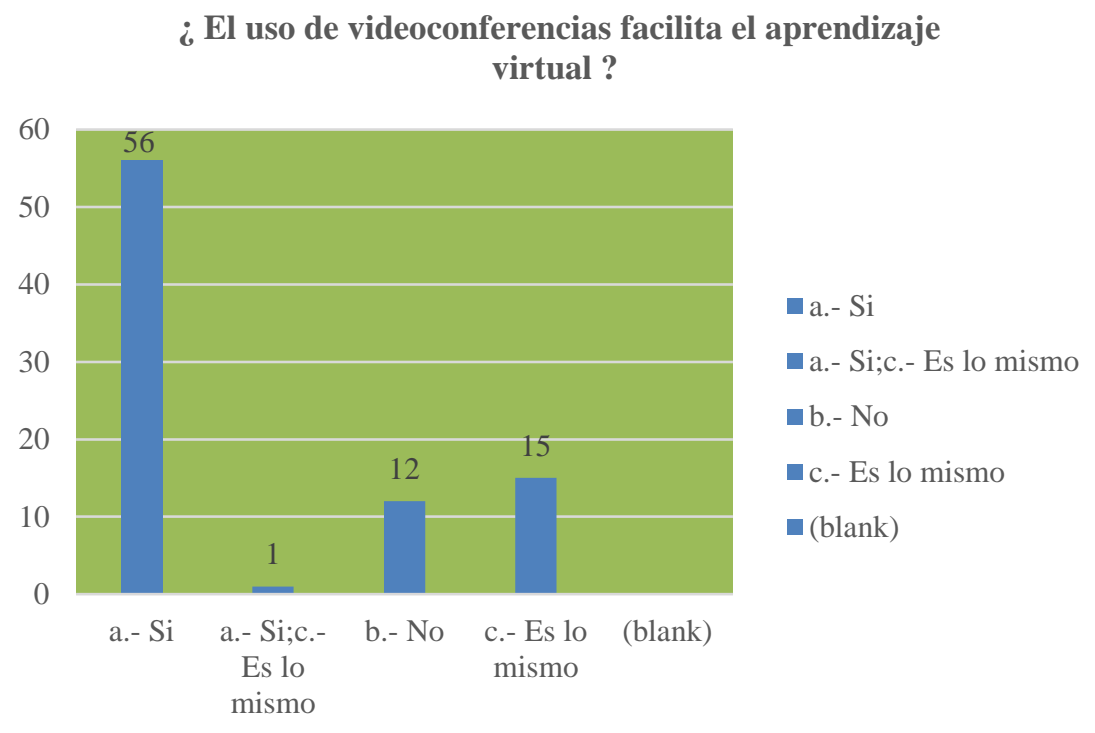

Fuente de consulta: "Elaboración Propia"

En el gráfico 7.6, 57 de los estudiantes encuestados consideran que sus competencias se desarrollarían mejor por videoconferencia, lo cual brinda una perspectiva similar al aprendizaje presencial, al utilizar la videoconferencia como medio de comunicación entre docentes y estudiantes, se continúa con la misma metodología de aprendizaje, teniendo como única variante el espacio, que en vez de agrupar a los estudiantes en una aula de clase, esta se migra al espacio virtual.

Gráfico 7.7 Estructura de los cursos en línea emergentes

Características de los cursos en línea según los encuestados

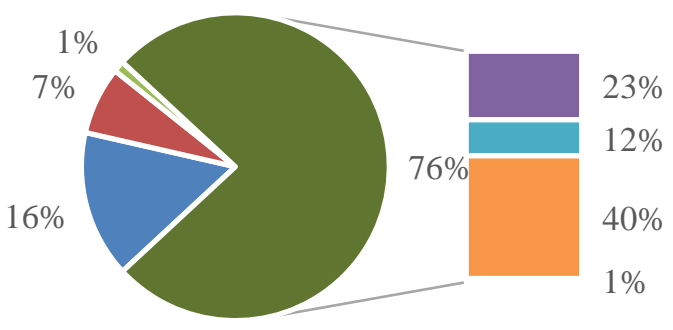

- a.- Incluye instrucciones, ejemplos, material de apoyo como bibliografía, videos del maestro explicando el tema, chat.

- a.- Incluye instrucciones, ejemplos, material de apoyo como bibliografía, videos del maestro explicando el tema, chat.;b.- Incluye instrucciones de la tarea y material de apoyo como videos de youtube, bibliografía;c.- Incluye la instrucción de la tarea y f
a.- Incluye instrucciones, ejemplos, material de apoyo como bibliografía, videos del maestro explicando el tema, chat.;c.- Incluye la instrucción de la tarea y fecha de

entrega
b.- Incluye instrucciones de la tarea y material de apoyo como videos de youtube, bibliografía

- b.- Incluye instrucciones de la tarea y material de apoyo como videos de youtube, bibliografía;c.- Incluye la instrucción de la tarea y fecha de entrega

\section{Fuente de consulta: "Elaboración Propia"}

De las respuestas obtenidas En el gráfico 7.7, sólo el 16\% de los estudiantes consideran que la estructura de su curso incluye: ejemplos, bibliografía para consultar, videos de maestros explicando el tema, chat, instrucciones de la tarea y material de apoyo como videos de youtube, y fecha de entrega; el $76 \%$ de los encuestados mencionan que no se les proporcionan ejemplos para llevar a cabo sus actividades, por lo que es muy importante que la instrucción por parte del docente se defina claramente para que el alumno logre captar lo más objetivamente posible lo que un autor ha querido transmitir a través de un texto escrito. 
Gráfico 7.8 Curso ideal según la perspectiva del estudiante

Caracterísiticas de un curso en línea consideradas por los encuestados

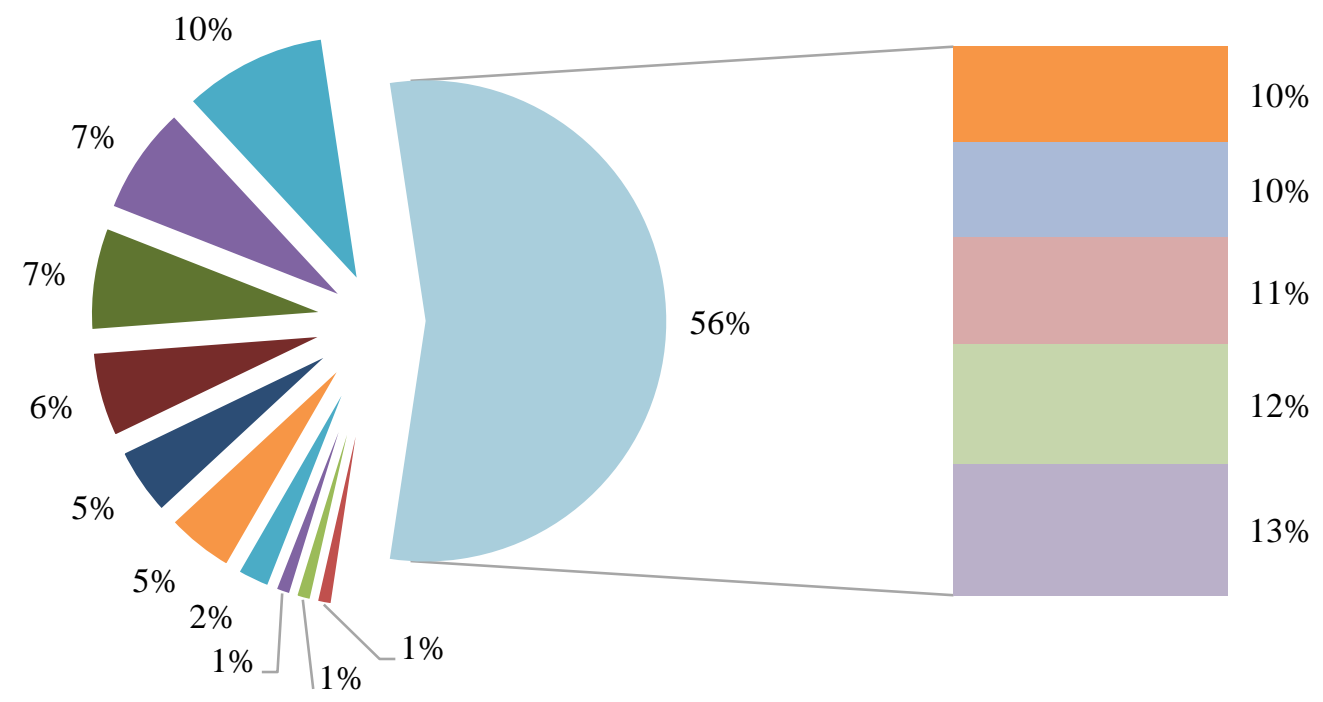

- a.- Que aparte de solo poner las instrucciones de la tarea incluya una introducción a la misma;d.- Recursos didácticos divertidos

- a.- Que aparte de solo poner las instrucciones de la tarea incluya una introducción a la misma;c.- Que usen material didáctico elaborado por el docente;d.- Recursos didácticos divertidos

- a.- Que aparte de solo poner las instrucciones de la tarea incluya una introducción a la misma;b.- Que cuando se envía una actividad sea retroalimentada por el maestro y nos regrese comentarios y calificación;d.- Recursos didácticos divertidos

- d.- Recursos didácticos divertidos

- a.- Que aparte de solo poner las instrucciones de la tarea incluya una introducción a la misma;b.- Que cuando se envía una actividad sea retroalimentada por el maestro y nos regrese comentarios y calificación;c.- Que usen material didáctico

- alaborado por e didáctico elaborado por el docente

- b.- Que cuando se envía una actividad sea retroalimentada por el maestro y nos regrese comentarios y calificación;c.- Que usen material didáctico elaborado por el docente;d.- Recursos didácticos divertidos

b.- Que cuando se envía una actividad sea retroalimentada por el maestro y nos regrese comentarios y calificación;d.Recursos didácticos divertidos

a.- Que aparte de solo poner las instrucciones de la tarea incluya una introducción a la misma

a.- Que aparte de solo poner las instrucciones de la tarea incluya una introducción a la misma;b.- Que cuando se envía una actividad sea retroalimentada por el maestro y nos regrese comentarios y calificación

- a.- Que aparte de solo poner las instrucciones de la tarea incluya una introducción a la misma;b.- Que cuando se envía una actividad sea retroalimentada por el maestro y nos regrese comentarios y calificación;c.- Que usen material didáctico

b.-- Que cuando se envía una actividad sea retroalimentada por el maestro y nos regrese comentarios y calificación;c.- Que usen material didáctico elaborado por el docente

b.- Que cuando se envía una actividad sea retroalimentada por el maestro y nos regrese comentarios y calificación

c.- Que usen material didáctico elaborado por el docente

Fuente de consulta: "Elaboración Propia" opciones:

En el gráfico 7.8, el 56\% de los resultados tiene una coincidencia en la selección de las siguientes

a. Que aparte de sólo poner las instrucciones de la tarea incluya una introducción de la misma

b. Que cuando se incluya una actividad sea retroalimentada por el maestro

c. Que usen material didáctico elaborado por el docente 


\section{d. Recursos didácticos divertidos}

Gráfico 7.9 Tipo de actividades de los cursos emergentes

\section{Porcentaje de actividades lúdicas y variadas que los estudiantes \\ detectaron}

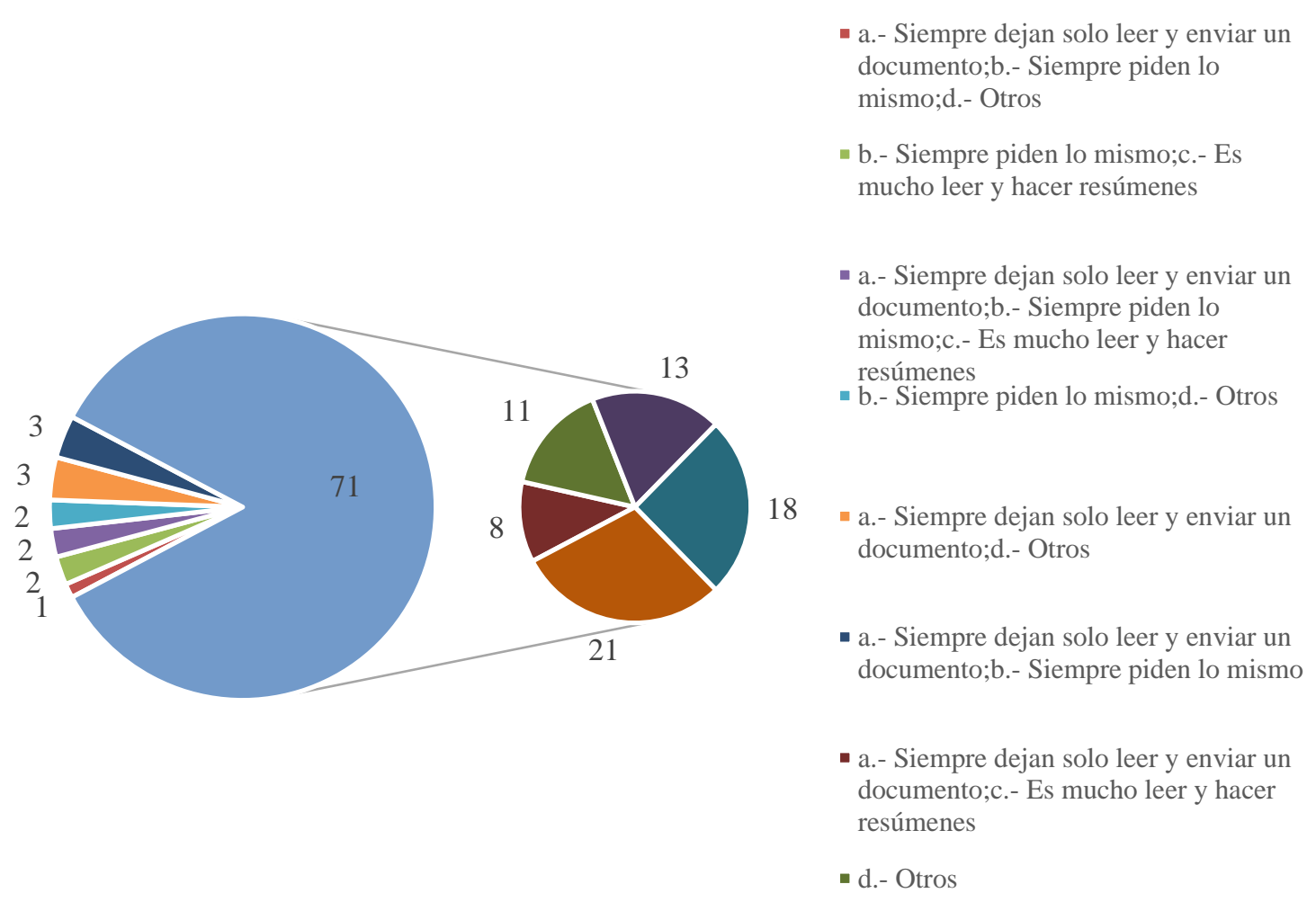

Fuente de consulta: "Elaboración Propia"

En el gráfico 7.9, los resultados seleccionados con mayor frecuencia por los estudiantes oscilan en las siguientes categorías:
a. Siempre dejan sólo leer y enviar un documento
b. Siempre piden lo mismo
c. Es mucho leer y hacer resúmenes

Con base en los resultados de los gráficos: 3,6 , y 9, podemos identificar que los estudiantes encuestados cuentan con la facilidad de conectarse a internet, lo cual no es de extrañarse ya que estamos inmersos en una sociedad del conocimiento, en donde las Tecnologías de Información y Comunicación se han convertido en un medio de contacto e interrelación entre las personas, ya sea por cuestiones de ámbito laboral, recreativo, o educativo, entre otros.

Sin embargo, cuando un estudiante que no ha experimentado el proceso de aprendizaje de forma virtual, aún teniendo las competencias digitales, y siendo un usuario frecuente de la tecnología busca de manera inconsciente que la metodología en línea sea similar a la presencial, lo cual claramente podemos afirmar con los resultados obtenidos en el gráfico 6. Entonces, una nueva pregunta que surgiría es: ¿Qué tipo de herramientas tecnológicas y proceso metodológico se requieren para estos estudiantes?

Es claro, considerando las respuestas de la pregunta 9, el estudiante encuentra monótona la clase, ya que en las actividades las instrucciones más frecuentes son leer y hacer resumen, entonces, una solución a esta premisa sería implementar actividades con ayuda de la tecnología, que promuevan la interacción y aprendizaje de los estudiantes, en donde su ambiente sea agradable y de fácil integración, y comunicación. 
Gráfico 7.10 Competencias requeridas para Modalidad Virtual

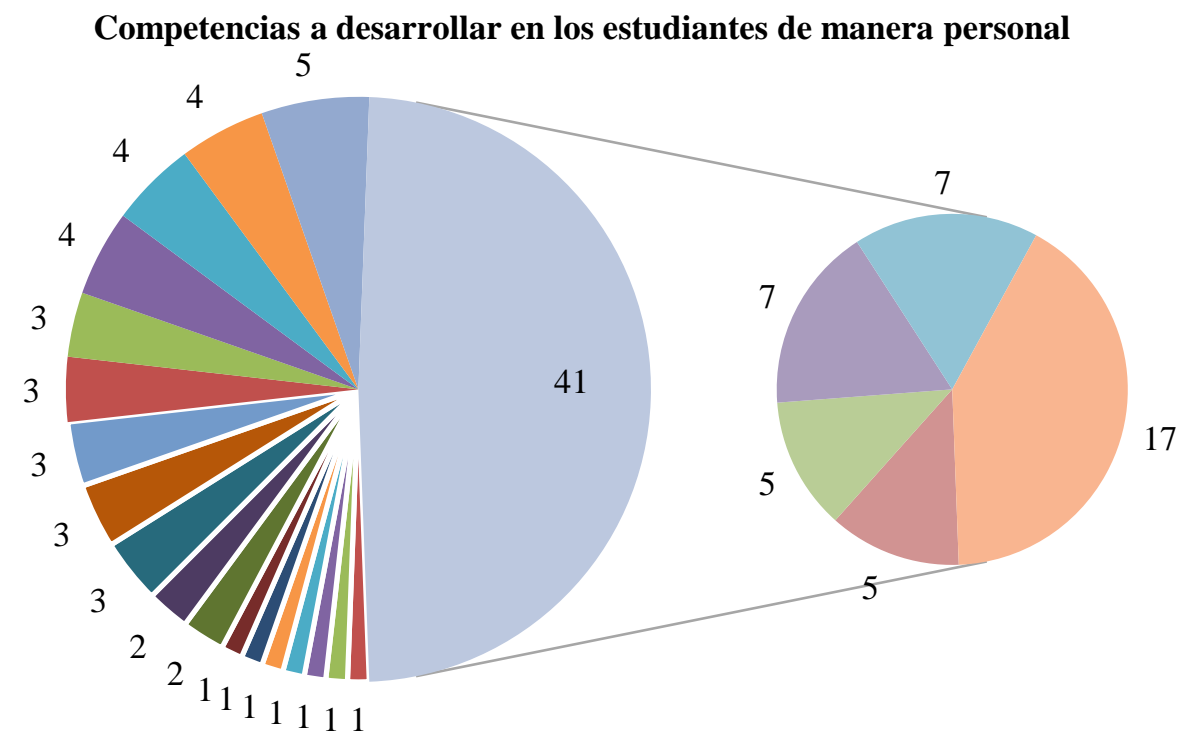

- a.- Administración del tiempo;c.- Compromiso;e.- Búsqueda de información de manera certera

- e.- Búsqueda de información de manera certera

- c.- Compromiso

- a.- Administración del tiempo;b.- Responsabilidad;d.- Disciplina;e.- Búsqueda de información de manera certera

- c.- Compromiso;d.- Disciplina

- d.- Disciplina

- c.- Compromiso;e.- Búsqueda de información de manera certera

- b.- Responsabilidad;d.- Disciplina;e.- Búsqueda de información de manera certera

- a.- Administración del tiempo;b.- Responsabilidad;d.- Disciplina

- b.- Responsabilidad;c.- Compromiso;e.- Búsqueda de información de manera certera

- f.- Ninguna

- a.- Administración del tiempo;c.- Compromiso

v.- Responsabilidad;c.- Compromiso

a.- Administración del tiempo;c.- Compromiso;d.- Disciplina

a.- Administración del tiempo;d.- Disciplina

a.- Administración del tiempo;d.- Disciplina;e.- Búsqueda de información de manera certera

a.- Administración del tiempo;b.- Responsabilidad;c.- Compromiso;e.- Búsqueda de información de manera certera

$\square$ a.- Administración del tiempo;b.- Responsabilidad;c.- Compromiso

$\square$ a.- Administración del tiempo;b.- Responsabilidad;e.- Búsqueda de información de manera certera

a.- Administración del tiempo;b.- Responsabilidad;c.- Compromiso;d.- Disciplina

Fuente de consulta: "Elaboración Propia"

En el gráfico 7.10, se muestran las competencias seleccionadas por los estudiantes que consideran necesarias para llevar a cabo su proceso de aprendizaje en línea, dentro de las más frecuentes se encuentran las siguientes:
a. Administración del tiempo
b. responsabilidad
c. Compromiso 


\section{d. disciplina}

Es imperante diferenciar entre la dinámica de aprendizaje de un ambiente presencial a uno virtual, ya que un estudiante que ha seleccionado la modalidad virtual, es aquel en el que éste es gestor de su propio aprendizaje, como lo indica Salamanca P.F. (2013), el estudiante se responsabiliza de su rol como tal, no se encuentra supeditado a un espacio y tiempo determinado, en el cual el profesor es el emisor de información y el estudiante el receptor. Es aquí donde ha sido complicado para el estudiante ya que la dinámica pedagógica a la que estaba acostumbrado ha cambiado inesperadamente. Es por ello, como lo indica el gráfico 10, que una de las competencias a desarrollar en los estudiantes, es la responsabilidad, de lo cual los encuestados en esta investigación son conscientes. Un estudiante de modalidad virtual es autónomo para adquirir y compartir conocimiento, y es su responsabilidad gestionar su propio aprendizaje con la guía del docente y haciendo uso de herramientas tecnológicas que le permitan lograr el objetivo de aprendizaje deseados.

Dos más de las competencias que requieren desarrollar los estudiantes además de la responsabilidad analizada en el gráfico 10, y de la administración del tiempo analizada con los resultados del gráfico 5, tenemos también las de índole digital y la autogestión.

Es muy interesante haber obtenido los resultados de la gráfica 5, en la cual los estudiantes son también conscientes de que esta nueva modalidad de estudios les demanda la modificación de la estructura pedagógica a la que estaban acostumbrados.

Siguiendo con Salamanca P.F. (2013), la educación a distancia fomenta la autonomía e independencia del estudiante, en donde éste autogestiona su proceso de aprendizaje, identificando y seleccionando herramientas que mayor beneficien al logro de los objetivos especificados de los diversos cursos en los que está inscrito. Es relevante que el estudiante reconozca cuales son sus debilidades académicas en esta nueva modalidad educativa en la que ha incursionado, y es por ello que necesita formalizar la dinámica de la autogestión para desempeñarse exitosamente en el área académica, así como tener la pre disponibilidad de aprender y utilizar herramientas tecnológicas que sirvan de ayuda en el logro de sus objetivos educativos, por lo menos durante el tiempo que dure la contingencia ocasionada por COVID-19.

\subsection{Agradecimiento}

Agradecemos al Comité organizador del CIERMMI 2020 y la empresa ECORFAN-MÉXICO por la invitación a participar en este programa de apoyo a profesoras-investigadoras, es un honor que se nos haya tomado en cuenta.

También agradecemos a los estudiantes que nos apoyaron con la respuesta al instrumento aplicado, gracias a ellos se pudo llevar a cabo este proyecto.

\subsection{Conclusiones}

De acuerdo con los resultados y con el objetivo planteado, concluimos que aún existe un gran abismo entre la modalidad de aprendizaje presencial y la modalidad de aprendizaje virtual. Se logró observar que la edad es una premisa y factor importante en la educación virtual debido a que los estudiantes en educación a distancia forman grupos heterogéneos en edad, intereses, ocupación, motivaciones, experiencias y aspiraciones, las competencias con las que cuentan son: Autorregulación del aprendizaje, Responsabilidad, Motivación, Autodisciplina, Metas bien definidas, Disposición, Autogestión, Búsqueda de Información, Administración del tiempo y Competencias digitales.

Los estudiantes en modalidades presenciales se caracterizan por ser en la mayoría de los casos grupos homogéneos, son interactivos, espontáneos, inquietos, resueltos, críticos, hábiles en el uso de tecnologías y ávidos de experiencias y sensaciones nuevas; pero están acostumbrados a que las indicaciones que reciben, así como la respuesta a sus dudas, se llevan a cabo por medio de su entorno académico, que es el aula presencial o también conocido como salón de clases. 
Confirmamos entonces la habilidad de los estudiantes en el campo tecnológico ya que éste no fue un obstáculo en relación a la conectividad, y tampoco en relación a la manipulación de las herramientas brindadas; la mayoría de los encuestados expresaron tener conectividad en sus domicilios por lo que no fue problema establecer y gestionar la comunicación, tanto con el docente como con sus compañeros.

Sin embargo, uno de los problemas que sí se detectó al analizar las respuestas de los encuestados, es la apreciación que estos tienen sobre la metodología de aprendizaje en ambientes virtuales en relación a la establecida a través de la modalidad presencial, la mayoría considera que de manera virtual no se logran los aprendizajes deseados, a pesar de que piensan que han hecho su mayor esfuerzo al cambio abrupto que tuvieron en su estilo de vida y aprendizaje.

Se observó que la modalidad de aprendizaje virtual es tan demandante como la modalidad presencial, ya que los encuestados mencionaron que le dedican más de cuatro horas diarias a la elaboración de tareas y a conectarse con el docente. En este punto se debe acentuar que los estudiantes encuestados no están acostumbrados a ser autogestivos y probablemente tampoco cuentan con un buen desarrollo de sus habilidades de lecto comprensión, consideran que si las clases se llevan por videoconferencia sí lograrían desarrollar las competencias requeridas, lo cual sería equivalente a una clase presencial; estableciendo comunicación sincrónica con el docente, quien explica el tema y les brinda acompañamiento durante del tiempo que dura la clase.

Estamos de acuerdo, que debido a la evidente etapa por la que atravesaron, tanto docentes como alumnos, la metodología pedagógica no fue la más adecuada para promover y gestionar el aprendizaje en el alumnado; como evidencia se obtuvo un gran porcentaje de respuestas en donde la labor del docente se limitó a dejar tareas sin establecer desde un principio un canal de comunicación asertivo, y sin recomendaciones para consultar recursos de apoyo, como por ejemplo, bibliografía que permitiera a los estudiantes realizar sus actividades adecuadamente.

El proceso de comunicación durante este período de contingencia se limitó de acuerdo a un gran porcentaje de los encuestados, a leer y hacer un resumen en la mayoría de las actividades, considerando el desarrollo de la actividad como aburrido.

Por lo anterior, los estudiantes consideran que los cursos deben de contener suficiente material de apoyo como videos del profesor explicando el tema, video tutoriales de youtube, material bibliográfico, ejemplos y algo muy importante, las instrucciones claras para que ellos puedan captar más objetivamente las competencias a desarrollar. Sugieren por otra parte que los cursos contengan material didáctico elaborado por el docente, así como recursos didácticos divertidos que ayuden al desarrollo de la competencia y puedan lograr un aprendizaje significativo.

Más de la mitad de los estudiantes encuestados manifestó que entre las competencias necesarias para realizar de manera satisfactoria el aprendizaje en la modalidad virtual, se encuentran: administración del tiempo, responsabilidad, compromiso y disciplina, las cuales son esenciales para su proceso de aprendizaje.

Asimismo, coincidimos en que las variables expuestas en esta investigación son relevantes para las estrategias de formación tanto de los docentes como de los estudiantes ya que, a mayor formación formal, el docente cree tener mayor competencia digital, competencia en la administración del tiempo, responsabilidad, compromiso y disciplina. Vale la pena mencionar que las dimensiones de estas competencias se relacionan tanto con docentes como con estudiantes, coinciden en la resolución de problemas; por ello, es importante reforzar la formación formal en ambos sentidos.

Esta contingencia fue el primer acercamiento para darnos cuenta que si bien se implementaron estrategias con el uso de las TIC para que los estudiantes continuarán con su proceso de aprendizaje, nos queda claro que la metodología que se emplea en la modalidad de aprendizaje presencial es diferente a la utilizada en la modalidad de aprendizaje virtual. Por consiguiente, es necesario desarrollar las competencias adecuadas para que tanto estudiantes como docentes se desempeñen exitosamente en un ambiente educativo no presencial. 
Es momento de que el Sistema Educativo implemente estrategias que fortalezcan la plantilla docente, para que sean motores de innovación y cambio metodológico al servicio de la mejora de los aprendizajes del alumnado (Hernández, González, Guzmán y Ordaz, 2016).

\subsection{Referencias}

Aguilar Peña, V.(2008). UNIVERSIDAD DE GUADALAJARA: RETOS Y PERSPECTIVAS EN EL SIGLO XXI. Guadalajara: Universidad de Guadalajara

Beatriz Zempoalteca Durán’Jorge Francisco Barragán LópezJJuan González Martínez ${ }^{\text {y } T e r e s a ~ G u z m a ́ n ~}$ Flores "Formación en TIC y competencia digital en la docencia en instituciones públicas de educación superior” Revista Apertura (Guadalajara, Jal.) versión On-line ISSN 2007-1094versión impresa ISSN 1665-6180 Apert. (Guadalajara., Jal.) vol.9 no.1 Guadalajara abr. 2017 http://dx.doi.org/10.32870/ap.v9n1.922

Brian Tracy (2016) Administración del tiempo, publicado en Nashville,Tennessee, Estados Unidos de América por Editorial Grupo Nelson.Inc es una subsidiaria que pertenece completamente a Thomas Nelson, Inc

Diario Oficial de la Federación https://dof.gob.mx/

Gobierno de México,Covid-19 https://coronavirus.gob.mx/covid-19/ recuperado el día 23 de mayo de 2020

Hernández, Juan; González, Juan; Guzmán, Teresa; Ordaz, Teresa. (2016). La Universidad Autónoma de Querétaro frente al reto de la formación de sus docentes: una reflexión sobre el modelo de competencia digital docente. Revista de Educación y Desarrollo, núm. 37, pp. 81-88.Recuperado http:www.cucs.udg.mx/revistas/edu_desarrollo/anteriores/37/37_HdzValerio.pdf

J. Casas Anguita, J.R. Repullo Labrador y J. Donado Campos (mayo 2003) La encuesta como técnica de investigación. Elaboración de cuestionarios y tratamiento estadístico de los datos (I) Revista Atención Primaria Vol. 31. Núm. 8. páginas 527-53

Ley General de Educación (2019), Cámara de Diputados del H. Congreso de la Unión. Recuperado el $\begin{array}{lllll}\text { día } & 25 & \text { de } & \text { mayo } & \end{array}$ http://www.diputados.gob.mx/LeyesBiblio/pdf_mov/Ley_General_de_Educacion.pdf

Moreno Almazán, Omar, \& Cárdenas López, María Georgina. (2012). Educación a distancia: nueva modalidad, nuevos alumnos. Perfiles de alumnos de Psicología en México. Perfiles educativos, 34(136), 118-136. Recuperado en 02 de junio de 2020, de http://www.scielo.org. $m x /$ scielo.php?script=sci_arttext\&pid $=$ S0185-

$26982012000200008 \& \ln g=e s \& \ln g=e s$.

Ponce Ponce María Enriqueta(2016) "La autogestión para el aprendizaje en estudiantes de ambientes mediados por tecnología" Diálogos sobre educación. Temas actuales en investigación educativa, vol. 7 , núm. 12, 2016 Universidad de Guadalajara, México DOI: https://doi.org/10.32870/ dse.v0i12.258

Pozos, Katia \& Mas, Oscar. (2012). The digital competence as a cross-cutting axis of higher education teachers' pedagogical competences in the European higher education area. Procedia-Social and Behavioral Sciences. vol. 46, pp. 1112-1116. http://dx.doi.org/10.1016/j.sbspro.2012.05.257

Ramírez, Alberto y Casillas, Miguel. (2014). Háblame de TIC. Tecnología digital en la educación superior. Argentina: Editorial Brujas-Socialtic. Recuperado de http://www.uv.mx/personal/albramirez/2014/08/01/hablamedetic

Real Academia Española, 2020, recuperado el dia 01 de junio 2020 https://dle.rae.es/responsabilidad?m=form 
Secretaría de Salud, comunicado 091 de fecha 18 de marzo de 2020 https://www.gob.mx/salud/prensa/091-reunion-de-trabajo-sobre-covid-19-entre-comites-nacionales-deemergencias-y-de-seguridad-en-salud?idiom=es recuperado el dia 23 de mayo de 2020

Romero-Mayoral, Jesús, \& García-Domínguez, Melchor, \& Roca-González, Cristina, \& Sanjuán Hernán-Pérez, Alejandra, \& Pulido-Alonso, Antonio (2014). DISEÑO DE UN APRENDIZAJE ADAPTADO A LAS NECESIDADES DEL ALUMNO. Teoría de la Educación. Educación y Cultura en la Sociedad de la Información, 15(3),172-189.[fecha de Consulta 2 de Junio de 2020]. ISSN: . Disponible en: https://www.redalyc.org/articulo.oa?id=2010/201032662010

Salamanca, P. F(2013). Entornos pedagógicos virtuales: acciones tutoriales con criterios de cobertura y calidad. ESPECÍFICAS EN IA DoCENCIA UNIVERSITARIA, 191.Didácticas específicas en la docencia universitaria Guillermo Londoño Orozco

WATHEN, Nadine; BURKELL, Jacquelyn. Believe it or not: factors influencing credibility on the web. Journal of The American Society for Information Science and Technology, v. 53, n. 2, p.134-144, 2002. Disponible en: http://onlinelibrary.wiley.com/doi/10.1002/asi.10016/pdf. https://doi.org/10.1002/asi.10016 